\title{
PUBLICACIONES SOBRE EVALUACIÓN DE LA ATENCIÓN PRIMARIA EN ESPAÑA TRAS VEINTE AÑOS DE REFORMA (1984-2004). ANÁLISIS TEMÁTICO Y BIBLIOMÉTRICO
}

\author{
Antonio de Lorenzo-Cáceres Ascanio $(1,2)$ y Ángel Otero Puime $(3,4)$ \\ (1) Centro de Salud Universitario "Ciudad Jardín". Servicio Madrileño de Salud. Madrid. \\ (2) Facultad de Medicina. Universidad Autónoma de Madrid (UAM). \\ (3) Departamento de Medicina Preventiva y Salud Pública. Facultad de Medicina. Universidad Autónoma de Madrid. \\ (4) Cátedra UAM / Novartis de Medicina de Familia y Atención Primaria.
}

\section{RESUMEN}

Ante la extendida opinión sobre la falta de datos para evaluar los resultados de la reforma de la Atención Primaria iniciada en España en 1984, se plantea realizar un análisis temático y bibliométrico de los trabajos de evaluación publicados. Se estudiaron los documentos publicados entre 1984 y 2004, localizados mediante búsqueda bibliográfica sistemática tanto en revistas biomédicas como en tesis, informes o libros, sobre cualquiera de los componentes de la Atención Primaria recogidos en la clasificación temática propuesta y referidos a ámbitos superiores a un solo centro de salud. Se utilizaron tres estrategias de búsqueda complementarias: bases de datos electrónicas, revisión manual de índices y búsqueda a partir de citas bibliográficas. 684 documentos cumplieron los criterios de inclusión. $85 \%$ procedían de revistas biomédicas, $12,3 \%$ eran tesis doctorales y $2,6 \%$ informes o libros. Se localizaron documentos en 49 revistas diferentes (29 extranjeras), siendo Atención Primaria $(52,7 \%)$ la que proporcionó más artículos. El $12,7 \%$ de los artículos fueron publicados en revistas indexadas en el SCI y un $8,6 \%$ en revistas extranjeras. En el $32 \%$ de los estudios el primer autor trabajaba en un centro de salud reformado. Dos tercios de las evaluaciones se centraron en el análisis del proceso. Los temas más frecuentemente evaluados se relacionaban con el gasto sanitario (farmacia), organización del sistema (urgencias) y satisfacción de los profesionales. Existe una amplia base documental con evaluaciones de la atención primaria española. Los estudios se han dirigido más hacia la evaluación del proceso que a la de resultados. La investigación en este campo requiere incluir trabajos no publicados en revistas (tesis e informes).

Palabras clave: Análisis bibliométrico. Atención primaria de salud. E.valuación. Reforma de los servicios de salud. España.

Correspondencia:

Antonio de Lorenzo-Cáceres Ascanio. Centro de Salud

Ciudad Jardín. C/ Pantoja, 3.28002 MADRID

Correo electrónico: antoniode.lorenzocaceres@uam.es

\section{ABSTRACT}

\section{Publications on Primary Care Evaluation in Spain after Twenty Years of Reform (1984-2004). Thematic and Bibliometric Analysis}

In view of the widespread opinion as to the lack of data for evaluating the results of the Primary Care reform which began in Spain in 1984, the conducting of a thematic and bibliometric analysis for the published evaluation studies is posed. The documents published within the 1984-2004 time period, located by means of systematic bibliographic search both in biomedical journals and in theses, reports or books on any of the components of Primary Care included within the proposed thematic classification and referred to scopes broader than one single healthcare center. Three complimentary search strategies were used: electronic databases, manual review of indexes and reference work quote-based searches. A total of 684 documents met the criteria for inclusion: $85 \%$ having been taken from biomedical journals, $12.3 \%$ having been doctoral theses, and $2.6 \%$ reports or books. Documents were located in 49 different journals (29 foreign), Atención Primaria (52.7\%) being the journal having provided the largest number of articles. A total $12.7 \%$ of the articles were published in journals indexed in the SCI, and $8.6 \%$ in foreign journals. In $32 \%$ of the studies, the first author was working at a reformed healthcare center. Twothirds of the evaluations revolved around the analysis of the process; the topics most often evaluated having been related to healthcare spending (pharmacy), the system organization (emergencies) and professional satisfaction. Broad-based documentation is provided with evaluations of primary care in Spain. Studies have been focused more on the evaluation of the process than on that of the results. Research in this field requires including studies not published in journals (theses and reports).

Key words: Bibliometric analysis. Primary Health Care. Evaluation studies. Assessment. Health care reform. Spain. 


\section{INTRODUCCIÓN}

En diciembre de 1984 se puso en marcha en España el proceso de reforma de la Atención Primaria de Salud (RAP). Todos los autores que han reflexionado sobre SUS $\log \operatorname{ros}^{1,2}$ o sus insuficiencias ${ }^{3,4}$ han expuesto sus valoraciones sobre los resultados concretos de la reforma. Pero en realidad, ¿qué es lo que sabemos sobre el impacto sanitario y en salud de la RAP española?

Los investigadores que se han interesado por el tema han coincidido en la necesidad de evaluar la RAP y, al mismo tiempo, en que faltan datos e investigaciones rigurosas. No dejan de reconocer la enorme dificultad de hacerlo ya que para ello sería preciso resolver previamente diferentes cuestiones. De una parte, es necesario delimitar la multiplicidad y diversidad de componentes, dimensiones y "productos" de la atención primaria que sería necesario eva$\operatorname{luar}^{5-10}$. Por otro lado, es conocida la dificultad de atribuir a las intervenciones de los servicios sanitarios los cambios observados en el nivel de salud de la población. A todo ello hay que añadir la ausencia de sistemas de información y de registros sistemáticos diseñados para tal fin y que incluyan datos del conjunto del territorio nacional a lo largo de estos veinte años. Puesto que en su momento no se establecieron estos sistemas de información y en la actualidad no es factible iniciar estudios retrospectivos para evaluar las transformaciones atribuibles a la RAP, la mayoría de los abordajes han quedado en opiniones personales más o menos fundadas en las conclusiones de un número limitado de estudios. La mayor parte de estos estudios evaluaban una única dimensión o producto de la Atención Primaria (AP) y, generalmente, se referían a ámbitos tan reducidos como un único Centro de Salud (CS). Es cierto que se han publicado comparaciones internacionales de varios indicadores de la AP española ${ }^{11-14}$ y evaluaciones generales relacionadas con servicios proporcionados por la $\mathrm{AP}^{15-16}$. También se han publicado un número reducido de estudios y documentos valiosos que evaluaban una o varias dimensiones de la RAP en ámbitos territoriales más amplios que un único centro de salud ${ }^{17-19}$. Todas estas circunstancias obligan a tratar de encontrar una metodología de estudio que permita abordar una evaluación de la RAP en su conjunto.

El objetivo de este trabajo es realizar un análisis temático y bibliométrico de las publicaciones aparecidas desde la reforma del modelo de Atención Primaria en 1984 y referidas a la evaluación de algún aspecto de la misma en un ámbito superior al de un único Centro de Salud.

\section{MATERIAL Y MÉTODOS}

Fuentes documentales: Se consideraron como posibles fuentes documentales los siguientes tipos de documentos: 1. Artículos publicados en revistas científicas sanitarias, tanto originales como cartas al director y otros (revisiones, artículos especiales,...). 2. Tesis doctorales presentadas en Universidades Españolas. 3. Informes de administraciones públicas, organismos, instituciones o sociedades científicas. 4) Libros.

Análisis temático: Para clasificar los temas de las distintas dimensiones, indicadores y tipos de resultado que permiten la evaluación de los componentes de estructura, proceso y resultado de la AP se confeccionó una clasificación temática ad hoc (sintetizada en el anexo 1) a partir de la propuesta de B. Starfield ${ }^{5}$ adaptada por Villalbí et $\mathrm{al}^{6}$ e incorporando algunos aspectos señalados por otros investigadores ${ }^{7-10}$. Uno de los autores clasificó los documentos para determinar el tema evaluado en cada trabajo. Cuando no fue posible identificar un tema principal, porque el estudio evaluaba más de un único componente o 
dimensión, se clasificó en el apartado de "evaluación de varios componentes".

Criterios de inclusión: Se seleccionaron los documentos o artículos que presentaban evaluaciones de cualquiera de los componentes de la AP recogidos en la clasificación temática propuesta realizados en más de un centro de salud, bien fuera en ámbitos geográficos superiores a una zona básica de salud (como una o varias áreas o distritos sanitarios, provincias, CCAA. o en toda España), o bien comparando centros entre sí (antes y después de la reforma, centros reformados frente a centros no reformados o centros reformados con distintas fórmulas de gestión y provisión de servicios).

Para evaluar la utilización que la AP realiza de la atención especializada y hospitalaria (como las interconsultas y urgencias hospitalarias) se utilizaron estudios realizados en el nivel especializado y hospitales siempre que se refiriesen a su ámbito geográfico de influencia, sin estudiar específicamente el comportamiento de un único centro de salud.

Las cartas al director fueron incluidas cuando aportaban datos sobre objetivos, metodología y resultados que cumplían los requisitos planteados en los criterios de inclusión de nuestro estudio. Se utilizaron los mismos criterios para incluir o no las comunicaciones a los Congresos de la Sociedad de Medicina de Familia (SEMFYC) publicadas en Atención Primaria hasta 1995 y a los Congresos de la Sociedad Española de Salud Pública y Administración Sanitaria (SESPAS) publicados en Gaceta Sanitaria hasta la actualidad. No se consideraron las comunicaciones publicadas en Libros de Actas distintos a las revistas y años ya mencionados.

Criterios de exclusión: Se excluyeron: 1. Estudios realizados en un único $C S$ o Zona Básica de Salud (ZBS). 2. Estudios en los que no fue posible recuperar, al menos, un resumen estructurado. 3. Estudios sin datos numéricos en el apartado de resultados. 4. Estudios en los que los resultados no responden a los aspectos de evaluación planteados en su título o en sus objetivos. 5. Artículos de opinión o debate. 6. Estudios duplicados. 7. Dada la estrategia de búsqueda utilizada se excluyeron los documentos referidos a evaluaciones de los siguientes aspectos de la AP: Docencia e investigación en AP, Servicios de promoción de la salud y de atención a la comunidad en AP. En el apartado de servicios asistenciales de la AP se excluyeron: atención pediátrica en AP: atención de enfermería en AP; servicios de matrona y de psicoprofilaxis obstétrica; servicios de fisioterapia y rehabilitación en AP; salud bucodental en AP y servicios de salud mental en AP.

Estrategias de búsqueda: Límite temporal: documentos publicados entre 1 enero de 1984 y el 31 de diciembre de 2004 , incluidos en las diferentes bases de datos antes del 1 de mayo de 2005, fecha en la que se realizó la última búsqueda. Lenguas: documentos en español, catalán o inglés. Descriptores o palabras clave: Para las búsquedas por medios electrónicos en bases de datos de revistas, de tesis y en archivos informatizados de bibliotecas se han utilizado lo siguientes términos MeSH (Medical Subject Heading): Primary Health Care / Primary Care / Atención Primaria; General Practice / Medicina General; Family Medicine / Family Practice / Family Physician / Medicina de Familia; Ambulatory Care / Atención Ambulatoria; Reform / Reforma; Spain / España; Assessment / Evaluation / Evaluación; Outcomes / Results / Resultados; Efficacy / Eficacia; Effectiveness / Efectividad; Efficiency / Eficiencia.

1) Búsqueda de artículos en revistas biomédicas: En aras de una mayor exhaustividad se utilizaron tres estrategias de 
búsqueda complementarias ${ }^{20}$ : A- Búsqueda en bases de datos electrónicas: bases documentales de Index Medicus-Medline (a través de Pub-Med y de Silver-Platter ERL), Science Direct, OVID y Colaboración Cochrane. B- Para los años y revistas no recogidos en las anteriores bases de datos electrónicas se realizó una búsqueda por revisión manual de los índices de las siguientes revistas: Atención Primaria, Medicina Clínica (Barc), Revista Española de Salud Pública (denominada Revista de Sanidad e Higiene Pública entre 1984 y 1995), Gaceta Sanitaria (hasta 1987 Gaseta Sanitaria de Barcelona), Medifam Revista de Medicina Familiar (1991-2004), Cuadernos de Gestión para el profesional de AP (19952004) y Revista de Administración Sanitaria (RAS) (1997-2004). C- Búsqueda a partir de las citas bibliográficas: Se seleccionaron manualmente citas bibliográficas de los documentos primarios cuyos títulos fueron considerados relevantes y no habían sido localizadas previamente mediante las estrategias de búsqueda electrónica y revisión manual de índices mencionadas anteriormente ${ }^{20}$. Buena parte de los artículos e informes publicados en ámbitos no sanitarios, fundamentalmente económicos, se localizaron gracias a este método.

Se consideró oportuno realizar una búsqueda electrónica en el Índice Medico Español (IME), a través del IMDOC del CSIC, pero finalmente se decidió no utilizarla ya que la consulta en línea del IME presentaba serias limitaciones al no permitir la consulta de un resumen estructurado para conocer el contenido del artículo, no disponer de tesauro normalizado y porque desde 1991 no se indexan la mitad de las revistas ni las cartas al director.

2) Búsqueda de tesis doctorales en universidades españolas: Se consultaron, mediante los términos Mesh ya citados, las siguientes bases de datos electrónicas de tesis doctorales españolas: 1) TESEO: Tesis presentadas en Universidades Españo- las (http://www.mcu.es/TESEO/teseo.html). 2) Unidad de Medicina Familiar de la Universidad de Sevilla: Tesis realizadas sobre Atención Primaria y Medicina de Familia en España (http://www.cica.es/aliens/ umfus/). 3) TDR - CBUC-CESCA: Servidor de Tesis Doctorales en Red del Consorci de Biblioteques Universitàries de Catalunya (http://www.tdr.cesca.es/index_ tdx_cs.html). Se han revisado todas las tesis doctorales sobre economía de la salud reseñadas en la web de la revista "Economía y Salud" (http://www.aes.es/publicaciones?pid=3\#Sumarios).

3) Búsqueda de informes de organismos e instituciones. libros: Se trataron de localizar todos aquellos documentos publicados en forma de informes por las diferentes administraciones sanitarias (autonómicas y central) conocidos a través de su cita en los documentos de referencia. Se utilizan los informes completos, libros o memorias cuando han podido ser recuperados o consultados en la Biblioteca Nacional de Ciencias de la Salud (BNCS) del Instituto de Salud Carlos III (ISCIII) o por cualquier otro medio que permitiera su consulta a los autores.

Etapas de la búsqueda y selección: Los documentos se seleccionaron en primer lugar a partir de las palabras clave en las búsquedas electrónicas, y a partir del título en las búsquedas manuales de los índices de las revistas. Una segunda selección se realizó a partir de la lectura de los resúmenes, o del documento completo cuando el resumen no permitía clarificar si se cumplían o no los criterios de inclusión.

Análisis bibliométrico: se utilizó la metodología recomendada por López Piñero ${ }^{21}$ que viene siendo utilizada en la mayoría de los estudios bibliométricos de trabajos biomédicos publicados en nuestro país, tanto en AP como en otras disciplinas $^{22-24}$. Entre otros indicadores se estudiaron las zonas de productividad de Brad- 
ford $^{21}$, para distribuir las revistas según su contribución al total de artículos seleccionados, y la adecuación a la ley de crecimiento de Price ${ }^{24}$, mediante la distribución en el tiempo de los artículos de esta disciplina. No se calcularon algunos de los indicadores de estudios bibliométricos clásicos, en particular la gráfica de la ley de Lotka o el análisis pormenorizado del consumo de información ${ }^{25}$ por considerar que excedían de los objetivos del trabajo.

Variables: Se confeccionó una base de datos Excel® con un registro para cada documento seleccionado con las variables referidas al documento, al primer autor, fuente documental o revista y consumo de información de interés para nuestros objetivos y utilizadas habitualmente en este tipo de estudios bibliométricos ${ }^{21,22,26}$. Se incluyeron además variables referidas al método de búsqueda por el que se localizó el documento.

\section{RESULTADOS}

Se revisaron inicialmente todos los documentos obtenidos mediante las estrategias de búsqueda descritas en el apartado de material y método. Así, de las 1.480 citas que en mayo de 2005 proporcionaba Medline sobre "APS y España", sólo 291 hacían referencia a aspectos de "evaluación". La revisión manual de índices de revistas biomédicas y la búsqueda a través de citas completó la selección hasta 582 artículos que cumplían los criterios de inclusión. Por su parte, la búsqueda del término "Atención Primaria" en la base TESEO proporcionó 386 tesis, de las que finalmente se incluyeron 84 . La búsqueda de libros e informes en la BNCS y por otros medios facilitó 18 referencias. En total para el estudio definitivo se seleccionaron 684 documentos.

Análisis de los temas: En la tabla 1 se presenta la distribución de los documentos según el tema de la AP evaluado. Dos ter- cios de los estudios evaluaron aspectos de la AP relacionados con el proceso $(66,5 \%)$. Los diferentes tipos de evaluación de resultado representaron un $16,1 \%$ y los trabajos que evaluaban la estructura de la AP el $14,7 \%$. Un $2,6 \%$ evaluaban más de uno de estos componentes al mismo tiempo. El tema más frecuentemente tratado fue el referido a la prescripción farmacéutica (20\%), tanto al gasto farmacéutico como a la calidad de la prescripción, seguido de la atención y derivación a hospitales de las urgencias (10\%). Otros temas se referían al clima laboral y satisfacción de los profesionales sanitarios $(7,3 \%)$, a la satisfacción de los usuarios $(6,7 \%)$, a la calidad de la atención de los procesos crónicos y a la derivación de pacientes entre la AP y la atención especializada (alrededor del 5,5\% cada uno).

\section{Estudio bibliométrico:}

1. Relativo a las fuentes documentales: El 85\% de los documentos seleccionados se encontraban publicados en revistas científicas, el $12,3 \%$ fueron tesis doctorales y el $2,6 \%$ era otro tipo de documentos ( $2 \%$ informes y monografías y un $0,6 \%$ libros).

Estrategias de búsqueda: Las búsquedas electrónicas en línea aportaron $28 \%$ de los documentos, de los que 5,7\% fueron localizados mediante el uso de Medline $\mathrm{y}$ $22,3 \%$ con otras bases de datos de acceso electrónico (fundamentalmente en las ediciones electrónicas de revistas españolas con acceso en línea). La revisión manual de los índices de las revistas clave se reveló como la estrategia de búsqueda más efectiva para localizar los documentos de interés en revistas biomédicas, al permitir la recuperación del $70 \%$ documentos seleccionados. Así, un 38,6\% de todas referencias se localizaron utilizando los índices en CD-ROM de las revistas y un $32 \%$ mediante la revisión manual número a número de las revistas en los años no recogidos en CD ROM. Finalmente, un 1,4\% de 


\section{FE DE ERRATAS}

En el trabajo publicado en el número 2 de 2007, Publicaciones sobre evaluación de la Atención Primaria en España tras veinte años de reforma (1984-2004). Análisis temático y bibliométrico. A de Lorenzo-Cáceres Ascanio y Á Otero Puime. Rev Esp Salud Pública 2007; 81: 131-145 existen dos erratas cuya corrección se presenta a continuación:

1. En la figura 2 debe aparecer la nota: La búsqueda no permitió conocer la institución de trabajo del primer firmante en todos los casos (ni 491).

2. La tabla 1 debe aparecer como sigue:

Tabla 1

Distribución de los estudios según el tema de evaluación de la AP y la fuente documental

\begin{tabular}{|c|c|c|c|c|c|}
\hline TEMA EVALUADO & $\begin{array}{c}\text { En revistas } \\
\text { (n) }\end{array}$ & $\begin{array}{c}\text { En tesis } \\
\text { (n) }\end{array}$ & $\begin{array}{c}\text { En otros } \\
\text { (n) }\end{array}$ & $\begin{array}{c}\text { Total } \\
\text { (N) }\end{array}$ & Porcentaje \\
\hline $\begin{array}{l}\text { - Estructura } \\
\text { - Proceso } \\
\text { - Resultado } \\
\text { - Varios componentes }\end{array}$ & $\begin{array}{r}87 \\
391 \\
91 \\
13 \\
\mathbf{5 8 2}\end{array}$ & $\begin{array}{l}14 \\
55 \\
15 \\
\mathbf{8 4}\end{array}$ & $\begin{array}{r}- \\
9 \\
4 \\
5 \\
18\end{array}$ & $\begin{array}{r}101 \\
455 \\
110 \\
18 \\
\mathbf{6 8 4}\end{array}$ & $\begin{array}{r}14,7 \\
66,5 \\
16,1 \\
2,6 \\
\mathbf{1 0 0 , 0}\end{array}$ \\
\hline $\begin{array}{l}\text { Farmacia } \\
\text { Urgencias } \\
\text { Clima laboral y satisfacción profesionales } \\
\text { Satisfacción usuarios } \\
\text { Calidad atención a pacientes crónicos } \\
\text { Derivación a atención especializada } \\
\text { Actividades preventivas } \\
\text { Resultados intermedios } \\
\text { Accesibilidad. Equidad de acceso } \\
\text { Uso de servicios (análisis de la demanda) } \\
\text { Eficiencia } \\
\text { Evaluación global de la AP (estructura, proceso, resultados) } \\
\text { Atención a pacientes terminales } \\
\text { Hospitalización por ACSC } \\
\text { Calidad general } \\
\text { Costes } \\
\text { Bajas Laborales (ILT) } \\
\text { Atención problemas alcohol } \\
\text { Estructura física y Recursos Humanos } \\
\text { Morbilidad cardiovascular evitada } \\
\text { Detección de factores de riesgo cardiovascular } \\
\text { Calidad de registros. Historia clínica } \\
\text { Cartera de servicios (oferta) } \\
\text { Mortalidad } \\
\text { Solicitudes de Laboratorio } \\
\text { Intervenciones sobre tabaquismo y hábitos tóxicos } \\
\text { Otros } 9 \text { temas (4docs. por tema o menos) }\end{array}$ & $\begin{array}{r}119 \\
65 \\
45 \\
38 \\
32 \\
34 \\
25 \\
26 \\
23 \\
20 \\
13 \\
13 \\
17 \\
10 \\
8 \\
11 \\
8 \\
8 \\
7 \\
6 \\
8 \\
7 \\
5 \\
5 \\
5 \\
5 \\
19\end{array}$ & $\begin{array}{r}20 \\
3 \\
5 \\
4 \\
5 \\
3 \\
2 \\
3 \\
6 \\
6 \\
4 \\
0 \\
1 \\
5 \\
6 \\
0 \\
1 \\
1 \\
1 \\
2 \\
0 \\
0 \\
1 \\
1 \\
0 \\
0 \\
4\end{array}$ & $\begin{array}{l}0 \\
1 \\
0 \\
4 \\
1 \\
0 \\
0 \\
5 \\
0 \\
0 \\
0 \\
0 \\
2 \\
5 \\
5 \\
0 \\
0 \\
0 \\
0 \\
0 \\
0 \\
0 \\
0 \\
0 \\
0 \\
0 \\
0 \\
0 \\
0 \\
0 \\
0 \\
0\end{array}$ & $\begin{array}{r}139 \\
69 \\
50 \\
46 \\
38 \\
37 \\
32 \\
29 \\
29 \\
26 \\
19 \\
18 \\
18 \\
15 \\
14 \\
11 \\
9 \\
9 \\
8 \\
8 \\
8 \\
7 \\
6 \\
6 \\
5 \\
5 \\
\mathbf{2 3}\end{array}$ & $\begin{array}{r}20,3 \\
10,1 \\
7,3 \\
6,7 \\
5,4 \\
5,3 \\
4,7 \\
4,2 \\
4,2 \\
3,8 \\
2,8 \\
2,6 \\
2,6 \\
2,2 \\
2,0 \\
1,6 \\
1,3 \\
1,3 \\
1,2 \\
1,2 \\
1,2 \\
1,0 \\
0,9 \\
0,9 \\
0,7 \\
0,7 \\
3,4\end{array}$ \\
\hline Total & 582 & 84 & 18 & 684 & 100,0 \\
\hline
\end{tabular}

ILT: Incapacidad Laboral Transitoria; ACSC: Ambulatory Care Sensitive Conditions. 
Figura 1

Año de publicación de los documentos seleccionados ( $\mathrm{n}=\mathbf{6 8 4}$ )

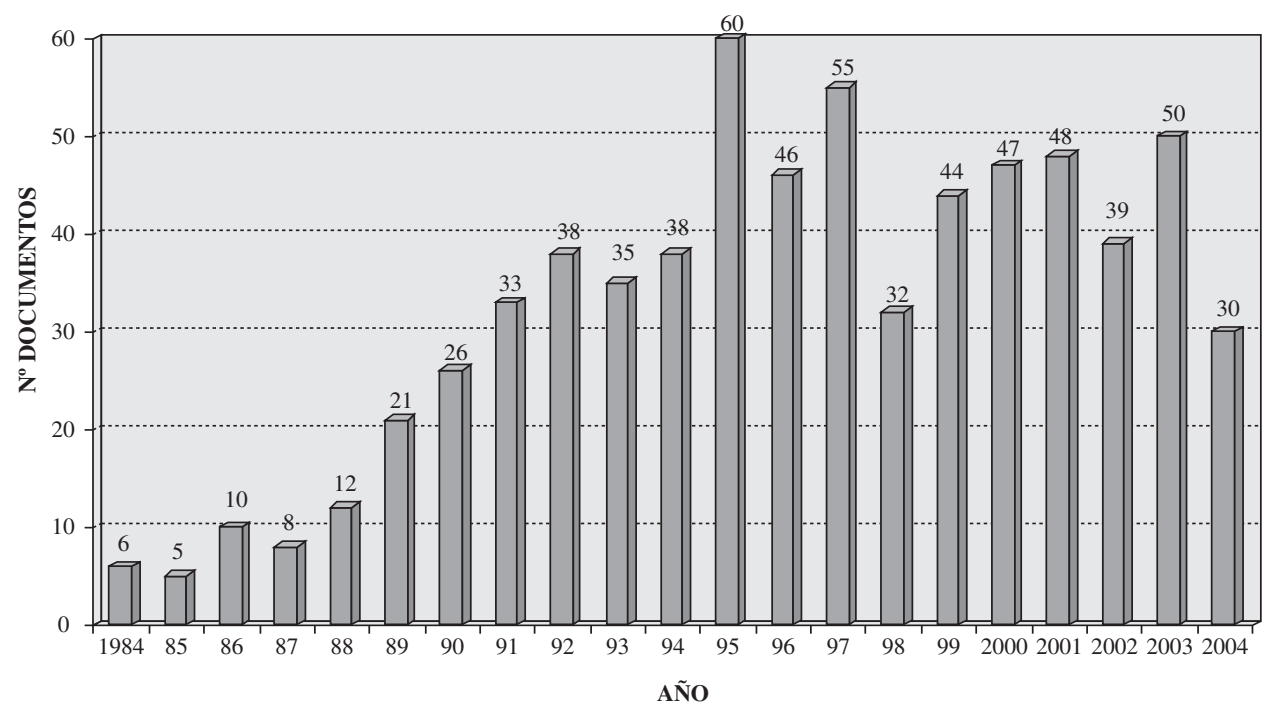

* NOTA: La búsqueda no permitió conocer la institución de trabajo del primer firmante en todos los casos (ni 491).

los documentos se localizó a partir de citas en otros documentos seleccionados.

En la figura 1 se indica la distribución de los documentos por año de publicación. La media de publicaciones por año fue de 32,8. En 1991 se alcanzó por primera vez esta cifra, situándose alrededor de 40 docs/año entre 1992-94 y en torno a unos 50 docs/año entre 1995 y 2003.

La practica totalidad de los documentos $(91,6 \%)$ estaba publicado en español, seguido por los documentos en inglés $(7,3 \%)$ y catalán $(1 \%)$.

Revistas: Se seleccionaron 582 documentos publicados en 49 revistas diferentes, de las que $29(56 \%)$ eran extranjeras, que publicaron $50(8,6 \%)$ de los documentos (tabla 1). La primera zona de Bradford la constituye la revista Atención Primaria con más de la mitad de los artículos seleccionados $(52,7 \%)$. El segundo nivel estaría constituido por las demás revistas, destacando Gaceta Sanitaria $(13,2 \%)$ y Medicina Clínica $(8,7 \%)$. Las revistas Medifam y Cuadernos de Gestión para el profesional de Atención Primaria, ya desaparecidas las

Tabla 2

Distribución por revistas de los documentos seleccionados

\begin{tabular}{|l|c|c|}
\hline \multicolumn{1}{|c|}{ REVISTAS } & $\begin{array}{c}\mathbf{N}^{\mathbf{0}} \text { de } \\
\text { Artículos }\end{array}$ & Porcentaje \\
\hline Atención Primaria & 307 & 52,7 \\
\hline Gaceta Sanitaria & 77 & 13,2 \\
\hline Medicina Clínica & 51 & 8,7 \\
\hline Medifam & 25 & 4,3 \\
\hline Cuadernos de Gestión en AP & 23 & 3,9 \\
\hline Rev Esp Salud Pública & 22 & 3,8 \\
\hline Otras 29 revistas extranjeras & 50 & 8,6 \\
\hline Otras 14 revistas españolas & 27 & 4,6 \\
\hline Total: 49 revistas & $\mathbf{5 8 2}$ & $\mathbf{1 0 0 , 0}$ \\
\hline
\end{tabular}


dos, y la Revista Española de Salud Pública (RESP) aportaron alrededor del $4 \%$ de los trabajos cada una. El resto de documentos apareció en revistas que publicaron tan sólo entre uno y cinco artículos cada una $(4,6 \%$ en otras 14 revistas españolas y $8,6 \%$ en 29 revistas extranjeras).

Un $80,1 \%$ de los artículos de revista seleccionados se publicaron como originales, el 9,9\% fueron comunicaciones a congresos publicadas en las revistas Atención Primaria y Gaceta Sanitaria, 6,4\% eran cartas al director y en el 3,5\% se trataba de otro tipo de artículos (revisiones, artículos especiales,...).

Visibilidad de los documentos seleccionados: El 12,7\% del total de artículos fueron publicados en revistas indexadas en el SCI con factor de impacto (FI). Dos tercios de estos artículos aparecieron en la revista Medicina Clínica. Aunque un 8,4\% de los artículos se publicó en revistas extranjeras, sólo la mitad de estos artículos fueron publicados en revistas indexadas en el SCI.

Tesis: Las tesis recuperadas y seleccionadas representaron el $12 \%$ del total de documentos de nuestro estudio y el $16 \%$ de todas las tesis sobre AP recogidas por TESEO en mayo de 2005. Entre 1986 y 2004 se presentaron una media de 4,4 tesisaño referidas a temas de evaluación de la AP. Los años más productivos fueron los del trienio 1997-99 con una media de 8 tesis-año. Por Comunidades, las Universidades de Madrid (24\%), Cataluña (16\%), Andalucía y Comunidad Valenciana (15 y $12 \%)$ son las más productoras. Por provincias, lo son las Universidades de Madrid, Barcelona, Murcia (8\%), Granada (7\%), Valencia y Alicante ( $6 \%$ cada una). La evaluación de la prescripción farmacéutica también fue el tema más frecuentemente tratado en las tesis (24\%), seguido del estudio del uso de los servicios y de la calidad de los mismos así como las desigualdades y la equidad en el acceso a estos servicios (7\% para cada uno de los tres temas).
Tabla 3

Productividad de los primeros autores de estudios de evaluación de la AP.

Número de documentos por autor $(*)$

\begin{tabular}{|l|c|c|c|}
\hline $\begin{array}{c}\text { Número de } \\
\text { documentos de } \\
\text { un mismo autor }\end{array}$ & $\begin{array}{c}\text { Número de } \\
\text { autores }\end{array}$ & $\begin{array}{c}\text { Total } \\
\text { documentos }\end{array}$ & \% \\
\hline 9 docs. & 1 & 9 & 1,3 \\
\hline 7 docs. & 1 & 7 & 1,0 \\
\hline 6 docs. & 3 & 18 & 2,6 \\
\hline 5 docs. & 3 & 15 & 2,2 \\
\hline 4 docs. & 10 & 40 & 5,8 \\
\hline 3 docs. & 77 & 51 & 7,4 \\
\hline 2 docs. & 379 & 379 & 55,4 \\
\hline 1 doc. & $\begin{array}{c}\text { Autor } \\
\text { colectivo }\end{array}$ & 8 & 1,2 \\
\hline- & $\begin{array}{c}\text { Autor } \\
\text { Anónimo }\end{array}$ & 5 & 0,7 \\
\hline- & $\mathbf{5 0 3}$ & $\mathbf{6 8 4}$ & $\mathbf{1 0 0 , 0}$ \\
\hline Total & & & 22,2 \\
\hline
\end{tabular}

(*) Datos referidos únicamente al primer autor.

Informes y Documentos: Se seleccionaron 13 informes y 4 libros para nuestro estudio.

2. Relativo a los autores: Los primeros autores/as de los 684 documentos representaron 503 autores diferentes. La distribución del número de documentos por primer autor se muestra en la tabla 3, oscilando entre un 55,4\% que firmaba un único documento como primer autor y un autor que firmó 9 documentos. La media de autores por artículo fue de 4,5 + 2,1 (4 de mediana y 4 de moda). El rango osciló entre un único autor $(5 \%)$ y 20 . Se localizaron 8 trabajos (1\%) firmados por un grupo de investigación y 5 Anónimos, realizados por organismos públicos. Para los propósitos de este trabajo no se analizaron los índices de productividad.

Instituciones: En los 491 (71\%) documentos (donde fue posible determinar el lugar de trabajo del primer autor (figura 2) 
Figura 2

Distribución de estudios según la institución de trabajo del primer autor

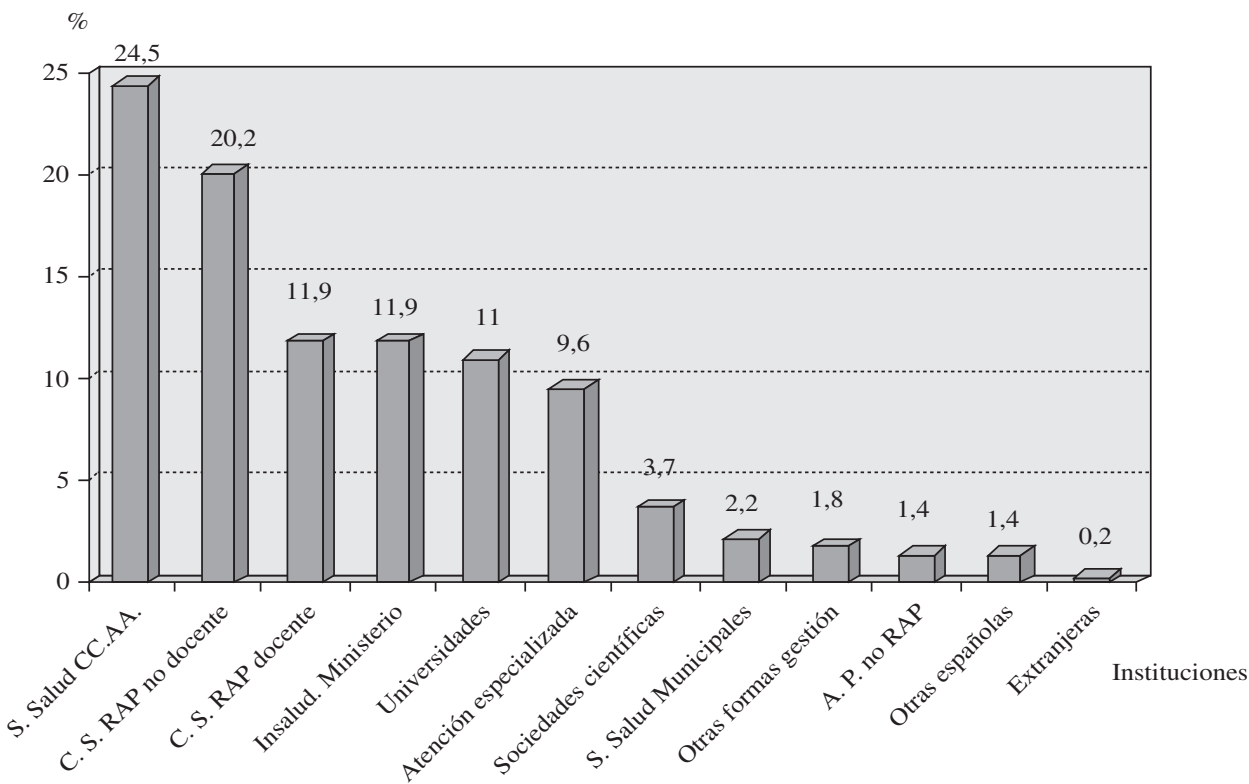

un $32 \%$ trabajaba en centros de salud del modelo reformado ( $20 \%$ en CS no docentes y $12 \%$ en CS docentes). El 24,5\% de primeros firmantes trabajaban en los Servicios de Salud de las CCAA, un $11,9 \%$ lo hacían en la Administración central del Estado (Insalud y Ministerio de Sanidad), otro $11,9 \%$ en Universidades españolas y un $9,5 \%$ en la Atención Especializada. Sólo dos trabajos eran firmados por autores de instituciones extranjeras.

Por CCAA, la mayoría de los trabajos procedían de Cataluña (29\%) seguida de Madrid (18\%), Valencia (10\%) y Andalucía $(8,9 \%)$. Las provincias de Barcelona $(27 \%)$ y Madrid (18\%) son las mayores productoras de documentos, seguidas de Valencia (4,9\%), Zaragoza, Alicante, Murcia y Oviedo con algo más de un $4 \%$ cada una (tabla 4).

3-Relativo al consumo de información: La media de citas por documento fue de
$14,4+13,3$ con un rango de $0-65$ citas por artículo.

\section{COMENTARIOS Y CONCLUSIONES}

La clasificación temática propuesta, que se resume en el anexo 1, trata de integrar y sistematizar las propuestas de diferentes autores sobre los distintos modos de abordar la evaluación de la atención primaria ${ }^{5-10}$.

En los veinte años transcurridos desde la puesta en marcha de la reforma de la AP en España, más de dos tercios de los estudios evaluaron el "proceso" de atención mientras que solamente el $16 \%$ eran evaluaciones de "resultados". Los temas más frecuentes se corresponden con aquellos de mayor influencia en el gasto sanitario (farmacia), con la organización y coordinación de los servicios asistenciales (utilización de las urgencias hospitalarias) o con aspectos concernientes a los propios profesionales (sa- 
Tabla 4

Procedencia geográfica de los primeros autores de los documentos. Distribución por Comunidades Autónomas y provincias

\begin{tabular}{|c|c|c|c|c|}
\hline \multirow{2}{*}{$\begin{array}{c}\text { Comunidades Autónomas y } \\
\text { provincias }\end{array}$} & \multicolumn{2}{|c|}{ Tesis } & \multicolumn{2}{|c|}{ Revistas } \\
\hline & n & $\%$ & $\mathbf{n}$ & $\%$ \\
\hline ANDALUCÍA & 13 & 15 & 52 & 8,9 \\
\hline - Granada & 6 & 7 & 23 & 3,9 \\
\hline ARAGÓN & 3 & 3,6 & 27 & 4,6 \\
\hline - Zaragoza & 3 & 3,6 & 26 & 4,5 \\
\hline ASTURIAS & 3 & 3,6 & 24 & 4,1 \\
\hline ISLAS BALEARES & - & - & 9 & 1,5 \\
\hline CANARIAS & 5 & 5,9 & 13 & 2,2 \\
\hline CANTABRIA & - & - & 4 & 0,6 \\
\hline CATALUÑA & 14 & 16 & 171 & 29,4 \\
\hline - Barcelona & 14 & 16 & 159 & 27,3 \\
\hline CASTILLA Y LEÓN & 3 & 3,6 & 24 & 4,1 \\
\hline CASTILLA-LA MANCHA & - & - & 15 & 2,6 \\
\hline EXTREMADURA & 1 & 1,2 & 11 & 1,9 \\
\hline GALICIA & 2 & 2,4 & 10 & 1,7 \\
\hline LA RIOJA & - & - & 6 & 1 \\
\hline MADRID & 20 & 24 & 107 & 18,4 \\
\hline MURCIA & 7 & 8 & 25 & 4,3 \\
\hline NAVARRA & 2 & 2,4 & 8 & 1,4 \\
\hline PAÍS VASCO & 1 & 1,2 & 19 & 3,2 \\
\hline C. VALENCIANA & 10 & 12 & 58 & 10 \\
\hline - Alicante & 5 & 6 & 26 & 4,5 \\
\hline - Valencia & 5 & 6 & 29 & 5 \\
\hline Total & 84 & 100 & $583 *$ & 100 \\
\hline
\end{tabular}

* NOTA: La búsqueda no permitió conocer la CC.AA. de procedencia en todos los casos.

tisfacción y clima laboral). A pesar de que la puesta en marcha de la reforma de la atención primaria supuso un considerable cambio organizativo, de manera que se llegó a hablar de un "nuevo modelo" frente al modelo "tradicional" anterior a la reforma, los estudios de evaluación se han dirigido hacia la gestión de los procesos más que a la evaluación de resultados en salud. El escaso número de estudios que evalúan los distintos tipos de "resultados" de la AP podría relacionarse con la reconocida dificultad para tratar de identificar y evaluar aquellos resultados imputables a la actividad de cualquier servicio sanitario.

Como en toda evaluación de servicios, la elección de los temas está influenciada por el interés de los diferentes agentes y por la facilidad de acceso a las fuentes de información disponible ${ }^{27}$. Así, la importancia que la factura farmacéutica tiene sobre el gasto imputable a los servicios de AP, su interés y facilidad de estudio para los responsables de gestión de la AP, al tratarse de datos registrados sistemáticamente y recogidos en soporte informático, la posibilidad de cruzar estos datos con las características del médico prescriptor y de la población adscrita, son factores que explicarían a nuestro juicio que el tema más frecuentemente evaluado fuese el relacionado con la prescripción farmacéutica en AP. Por otra parte, el crecimiento de la demanda de atención urgente de los últimos años se ha tratado de relacionar con la mayor o menor ineficacia de la AP para contener dicha utilización, lo que explicaría el interés para evaluar la utilización de los servicios de urgencia hospitalarios por parte de la población general.

Llama la atención que el análisis de la satisfacción de los profesionales parece interesar más que la evaluación de la satisfacción de los propios pacientes con los servicios prestados. Los temas de los estudios relacionados con los profesionales siguen una cierta distribución temporal a lo largo de los años. Si en un primer momento se analizaron factores relacionados con el trabajo en equipo, el clima organizacional y los conflictos de rol, en los últimos años cada vez con más frecuencia se repiten estudios que evalúan la satisfacción laboral y el desgaste profesional (burnout), lo que puede relacionarse con las expectativas más o menos insatisfechas de los profesionales de la AP. 
En cuanto al análisis bibliométrico, hasta principios de los años 80 la investigación en y sobre la AP española era testimonial en las publicaciones biomédicas. Entre 1971 y 1983 Marset $^{24}$ localizó en el IME tan sólo 109 trabajos relacionados con cualquier tema de AP. Nuestro trabajo, que estudia una pequeña parte de los estudios sobre la AP española, revela un crecimiento sostenido de estas publicaciones entre 1984 y 1991, estabilizándose a partir de 1992 entre 40 y 50 documentos-año. El ritmo de crecimiento de los trabajos parece ir paralelo a los cambios en la periodicidad de la revista Atención Primaria, aparecida en 1984 como publicación bimensual, pasando a mensual en 1987 y a quincenal en 1992. A partir del año 1995, año en el que se seleccionan 60 trabajos, se observó un estancamiento en el ritmo de crecimiento del número de trabajos-año, lo que incumpliría la ley de Price, algo que ya se ha descrito en otros estudios sobre la investigación general en $\mathrm{AP}^{24}$ y en otras especialidades ${ }^{22}$. Este dato, sumado al ya mencionado aumento en la frecuencia de estudios sobre el desgaste de los profesionales, podría sugerir un cierto agotamiento del interés por el modelo.

En nuestro trabajo, Atención Primaria es la publicación que concentra más de la mitad de los documentos localizados en revistas, lo que es congruente con el dato de que concentrase el 58\% de toda la investigación española sobre AP entre 1971 y $1992^{24}$ y el $78 \%$ entre 1988 y $1992^{23}$.

Pese al gran número de publicaciones localizadas, tan sólo el $12 \%$ de los artículos se publicó en revistas indexadas en el SCI. De todas ellas, Medicina Clínica es la revista con mayor repercusión, pues en ella aparecieron el 75\% de los artículos publicados en revistas con FI reconocido por el SCI. Creemos que este dato se relaciona con el prestigio de esta publicación entre los clínicos y la línea editorial de la revista para publicar trabajos de investigación realizados en nuestra AP.
El escaso número de trabajos publicados en inglés creemos que pone de manifiesto una limitación idiomática de los investigadores de AP. Nos resulta difícil explicar por qué la mitad de los trabajos publicados en el extranjero lo hicieron en revistas no indexadas en el SCI.

La experiencia de la reforma de la AP española parece haber despertado poco interés en el contexto internacional, aunque algunos informes supranacionales incluyen datos e indicadores de los cambios en la situación sanitaria de la AP España a lo largo de estos años ${ }^{11-14}$. La mínima presencia de autores extranjeros nos lleva a pensar que la evaluación de la AP española puede haber sido considerada por los editores de revistas y los autores no nacionales como un tema de interés local, circunscrito a nuestro país. La visibilidad de los artículos publicados se ha visto perjudicada por el reducido número de revistas biomédicas españolas incluidas en el SCI. Como ha apuntado un reciente estudio ${ }^{28}$, varias revistas españolas -incluida Atención Primaria- obtendrían un FI de tal magnitud que les permitiría estar incluidas en la cobertura del SCI. Pensamos que la reciente introducción de la RESP en el Social Science Citaron Index (SSCI) favorecerá la difusión internacional de los trabajos realizados sobre la AP española.

En la mayoría de los estudios sobre productividad científica en España se obtienen indicadores bibliométricos superponibles al que presentamos. La media de citas obtenida fue algo inferior a la hallada en otros estudios $^{22-23}$, al haberse incluido cartas y comunicaciones. La media de autores por artículo es similar a la hallada en otros estudios y disciplinas 22-23, 29. Barcelona, Madrid, Comunidad Valenciana y Andalucía son también las CCAA más productivas $^{22,26}$. Paralelamente, Barcelona y Madrid destacan como las provincias con más publicaciones ${ }^{30}$. Otros autores son partidarios de no utilizar indicadores absolutos para 
evaluar la productividad geográfica sino indicadores relativos en relación a la población provincial ${ }^{22-23}$, a la renta o al número de médicos de $\mathrm{APS}^{24}$, con lo que provincias como Oviedo, Murcia, Zaragoza o Navarra se colocarían entre los primeros lugares.

En orden a lograr la mayor exhaustividad en la revisión, un aspecto de interés en nuestra investigación ha sido la búsqueda realizada entre la denominada "literatura gris". Las tesis doctorales son parcialmente recuperables, al menos en forma de resumen estructurado, a través de la base de datos Teseo. Ello ha permitido localizar un número importante de tesis útiles para los propósitos de nuestro estudio. Pese a tratarse de documentos poco accesibles para la mayoría de los investigadores, fue necesario la consulta de informes y documentos de organismos oficiales de la administración del estado (central y autonómica) que evaluaban aspectos relacionados con el uso de servicios (memorias de los diferentes Servicios de Salud), la equidad (Encuestas de Salud), la satisfacción de los usuarios (encuestas de satisfacción, barómetros del CIS,..), etc.

Como limitaciones de nuestro trabajo cabe señalar que el análisis temático fue realizado por un único evaluador (ALC) y que se clasificó un único tema principal por trabajo. A diferencia de otros estudios bibliométricos, no se calcularon algunos de los indicadores bibliométricos clásicos, particularmente alguno de los relativos al consumo de información, y sólo se analizaron los datos referidos al primer autor de cada documento. Con todo, se ha podido confirmar una relación inversamente proporcional entre el número de primeros autores y el de documentos por autor (tabla 2), y la presencia -como en otras disciplinas- de un pequeño grupo de autores especialmente prolíficos. En otros estudios bibliométricos de temática más general se constata un alto índice de transitoriedad $\left(72 \% 26-82 \%{ }^{22}\right)$. Sin embargo, en nuestro estudio parece encon- trase una franca especialización de los autores que evalúan la AP española ya que tan sólo un 55,4\% de los primeros autores firmaba un único artículo.

En el apartado de métodos, ya se comentaron los motivos para no utilizar la búsqueda en línea realizada en el IME. Las limitaciones de esta base de datos se pusieron de manifiesto cuando una búsqueda inicial solamente señaló 103 referencias sobre "Evaluación y Atención Primaria". Por otro lado, aunque el IME permitía la localización de documentos relacionados con áreas temáticas poco representadas en las revistas seleccionadas para su revisión manual (como pediatría, enfermería,...), estos temas fueron excluidos finalmente de nuestro estudio, pese a considerarlos como parte importante de los servicios ofrecidos por la AP española.

Entre otros posibles sesgos, a la hora de analizar los resultados de la RAP publicados en cada artículo concreto, debería tenerse en consideración el posible sesgo de publicación que tendería a presentar para su publicación aquellos estudios que mostraran resultados favorables a la RAP y a no publicar aquellos con datos negativos.

Algunos autores proponen eliminar de los estudios bibliométricos las cartas al director $^{22}$. Otros, en cambio, consideran las cartas como artículos formales breves. En nuestro estudio consideramos que la información recogida durante los primeros años de la RAP adoptaba con frecuencia este formato, ya que era necesario evaluar experiencias aisladas o novedosas. Es preciso señalar que hasta el año 1991 no se generalizó el resumen estructurado en los artículos originales. En todo caso, la mayoría de los documentos publicados en revistas e incluidos en nuestro estudio, han sido artículos originales $(80 \%)$.

Aunque el $85 \%$ de los documentos seleccionados son recuperables para la mayoría 
de los investigadores, por haber sido publicados en revistas accesibles del ámbito de la AP y biomedicina, el diseño de nuestro estudio no permitía utilizar únicamente los medios de búsqueda electrónica. De hecho, la estrategia de búsqueda más productiva fue la búsqueda manual número a número de los índices de las revistas, bien a través de la consulta directa de los ejemplares o a través de bases de datos electrónicas (en soporte CD ROM, o con acceso a la versión en línea de las revistas), lo que limitaría la reproducibilidad del estudio.

En cuanto al tratamiento de las pérdidas, la naturaleza de algunas fuentes consultadas y alguno de los métodos de búsqueda no permitieron recuperar todas las variables de estudio en cada documento, por lo que algunos datos no se refieren al total de los documentos, principalmente cuando nos referimos a la institución de trabajo del primer autor y a la comunidad autónoma o provincia de residencia de estos.

En el futuro, las líneas de investigación debieran dirigirse al análisis de los diferentes indicadores de resultado de la AP. Las evaluaciones de mayor interés serían los estudios de efectividad (grado de resolución) y de eficiencia, en lugar de seguir analizando la calidad de proceso. Es necesario dirigir las publicaciones hacia revistas con mayores posibilidades de difusión entre la comunidad científica internacional, especialmente las indexadas en el SCI.

En conclusión, existe una abundante base documental que puede permitir la evaluación de la RAP en España. Pensamos que es posible componer una visión de conjunto mediante nuevos estudios que realicen análisis parciales con los documentos que han evaluado los diferentes componentes y dimensiones de la AP a lo largo de estos años. Para ello sería preciso profundizar en el análisis de la documentación localizada y seleccionada, empleando técnicas de revisión sistemática de la literatura con criterios explícitos de valoración de la calidad de los trabajos publicados.

\section{BIBLIOGRAFÍA}

1. Minué Lorenzo S, de Manuel Keenoy E, Solas Gaspar O. Situación actual y futuro de la atención primaria, en Cabasés JM, Villabí JR eds. Invertir para la salud. Prioridades para la salud pública. Informe SESPAS 2002. Disponible en URL http://www.sespas.es/fr_inf.html [citado el 13 de febrero de 2007.

2. Martín Zurro A, Ledesma Castellfort A, Sans Miret A. El modelo de atención primaria de salud: balance y perspectivas. Aten Primaria 2000: 25 (1): 48-58.

3. Comisión de análisis y evaluación del Sistema Nacional de Salud. Informe de la Subcomisión de Atención Primaria, en Informe y recomendaciones (Informe Abril). Madrid; 1991.

4. Ortún V, Gervas J. Fundamentos y eficiencia de la atención médica primaria. Med Clin (Barc) 1996; 106 (3): 97-102.

5. Starfield, B. A basis for evaluating Primary Care, en Primary Care. Concept, evaluation and policy. New York: Oxford University Press; 1992.

6. Villalbí JR, Pasarín M, Montaner I, Cabezas C, Starfield B y Grupo de Trabajo sobre Evaluación en la Atención Primaria de Salud de Barcelona. Evaluación de la Atención Primaria de Salud. Aten Primaria 2003; 31 (6): 382-5.

7. García Olmos L, Minué Lorenzo S. La medida del producto de la atención primaria. Cuadernos de Gestión para el profesional de Atención Primaria 1998; 4 (4): 191-200.

8. Plaza Tesías A, Guarda Rojas A, Farrés Quesada J, Zara Yanhi C y Grupo Asesor. Consenso sobre un proceso de benchmarking en la atención primaria de salud de Barcelona. Aten Primaria 2005; 35 (3): 130-9.

9. Badía Llach X, Lizán Tudela L. Reflexiones sobre la investigación de resultados en salud. Aten Primaria 2002; 30 (6): 388-91.

10. Jiménez Villa J, Cutillas Castell S, Martín Zurro A y Grupo MPAR-5. Evaluación de resultados en atención primaria: el proyecto MPAR-5. Aten Primaria 2000; 25 (9): 653-62. 
11. Macinko J, Starfield B. The contribution pf primary care systems to health outcomes within Organization for Economic Cooperation and Development (OECD) countries 1970-1998. Health Serv Res 2003; 38: 831-65.

12. Starfield B, Shi L. Policy relevant determinants of health: an international perspective. Health Policy 2002; 60 (3): 201-18.

13. Saltman RB, Rico A, Boerma WG. Primary Care in the driver,s set. Organizational reform in European primary care. Glasgow: Open University Press; 2006. Disponible en http://www.euro.who. int/Document/E87932.pdf, citado el 12 de febrero de 2007.

14. López Casasnovas G, Planas Miret I. Anàlisi dels models organitzatius europeus en 1,àmbit de 1,atenció primària. Fulls Econòmics 2003; 37: 48-54.

15. Ponsá JA, Cutillas S, Elías A, Fusté J, Lacasa C, Oliver $\mathrm{M}$ et al. Avaluació de la reforma de 1, atenció primària i de la diversificació de la provisió de serveis. Fulls Económics del Sistema Sanitari 2003; 37: 33-9.

16. Aguilera M, Alfaro M, Crespo B, Custodi J, Guerra J, Ichaso MS, Pérez JA, Ruiz AM (coords.). Atención Primaria en el INSALUD. 17 años de experiencia. Madrid: Instituto Nacional de la Salud; 2002.

17. Plaza Tesías A, Zara Yahni C, Guarda Rojas A, Farrés Quesada J. Resultado de la aplicación del benchmarking en los equipos de atención primaria de Barcelona. Aten Primaria 2005; 35 (3): 122-9.

18. Goñi S. An análisis of effectiveness of Spanish primary care teams. Health Policy 1999; 48 (2): 107-117.

19. Guarga A, Gil M, Pasarín M, Manzanera R, Armengol R, Sintes J. Comparación de equipos de atención primaria de Barcelona según fórmulas de gestión. Aten Primaria 2000; 26 (9): 600-6.

20. Abad MA, Aleixandre R, Peris R. Estrategia de búsqueda de artículos de revistas españolas. Estudio de un caso: evaluación de la calidad de los sistemas de información. Gac Sanit 1995; 9: 363-70.

21. López Piñero JM, Terrada ML. Los indicadores bibliométricos y la evaluación de la actividad médico-científica. (III) Los indicadores de pro- ducción, circulación y dispersión, consumo de la información y repercusión. Med Clin (Barc) 1992; 98: 142-8.

22. Álvarez Solar M, López González ML, Cueto Espinar A. Indicadores bibliométricos, análisis temático y metodológico de la investigación publicada en España sobre epidemiología y salud pública (1988-1992). Med Clin (Barc) 1998; 111: 529-535.

23. Álvarez Solar M, López González ML, Cueto Espinar A. Análisis temático y metodológico de la investigación en atención primaria (1988-1992). Aten Primaria 1996; 18: 297-303.

24. Marset Campos P, Sáez Gómez JM, Sánchez Moreno A, Ramos García E, Sánchez Estévez V, González Díaz M. Perspectiva de la atención primaria española a partir del análisis bibliométrico de su producción científica (1971-1994). Aten Primaria 1997; 19 (8): 389-94.

25. Aleixandre R, Jiménez Sánchez JV, Terrada Ferrandiz ML, López Piñero JM. Análisis del consumo de información en la revista Atención Primaria. Aten Primaria 1996; 17 (5): 321-5.

26. Aleixandre R, Porcel A, Agulló A, Marset S, Abad F. Diez años de la revista Atención Primaria (1984-1993): análisis bibliométrico y temático. Aten Primaria 1996; 17: 225-30.

27. Rossi PH, Lipsey MW and Freeman HE. Evaluation: A systematic approach ( $7^{\mathrm{a}}$ ed.). Thousand Oaks (Calif.): Sage Publications; 2004.

28. Aleixandre R, Valderrama JC, González de Dios J, de Granda JI, Miguel-Dasit A. El factor de impacto: un polémico indicador de calidad científica. Economía de la Salud 2004; 3: 242-9. Disponible en URL http://ime.uv.es/imecitas/impacto. shtml citado el 13 de febrero de 2007.

29. Pérez Andrés C, Estrada Lorenzo JM, Villar Álvarez F, Rebollo Rodríguez MJ. Estudio bibliométrico de los artículos originales publicados en la Revista Española de Salud Pública. I. Indicadores generales. Rev Esp Salud Pública. 2002; 76(6): 659-72.

30. Camí J, Suñén Piñol E, Méndez Vásquez R. Mapa bibliométrico de España 1994-2002: biomedicina y ciencias de la salud. Medicina Clínica 2005; 124 (3): 93-101. Corrección errata en: Med Clin (Barc) 2005; 124 (11): 414. 


\section{ANEXO 1}

\section{CLASIFICACIÓN TEMÁTICA DE LOS ESTUDIOS DE EVALUACIÓN DE LA ATENCIÓN PRIMARIA *}

\section{I) EVALUACIÓN DE LA ESTRUCTURA:}

Capacidad asistencial

1. Estructura física y Recursos humanos

2. Accesibilidad y Equidad de acceso

3. Continuidad y Longitudinalidad

5. Modelos de gestión

6. Clima laboral y satisfacción de los profesionales de AP

7. Cartera de servicios (catálogo ofertado)

II) EVALUACIÓN DEL PROCESO: Práctica asistencial

\section{SERVICIOS PRESTADOS POR LA AP (Outputs):}

a. Integralidad: Servicios asistenciales, preventivos y de promoción de la salud

b. Demanda y uso de servicios

c. Calidad de los servicios

2. CONSUMOS Y GASTOS GENERADOS:
a. Capacidad resolutiva y Coordinación: Derivaciones a consultas atención especializada y a urgencias hospitales
b. Laboratorio
c. Radiología y otras técnicas diagnósticas
d. Farmacia: gasto y calidad
e. Bajas laborales (ILT)
f. Costes generales y Eficiencia

\section{DOCENCIA. INVESTIGACIÓN}

III) EVALUACIÓN DE RESULTADOS: Cambios en la salud de la población.

1. EFECTIVIDAD: RESULTADOS EN SALUD (Outcomes):
a. Reducción de la mortalidad
b. Mejoras en el nivel de salud de la población. Salud autopercibida
c. Mejoras en la calidad de vida relacionada con la salud (CVRS)
d. Resultados intermedios:
- Reducción de factores de riesgo y de hábitos tóxicos
- Reducción de morbilidad prevenible y complicaciones evitables
- Control de enfermedades crónicas

e. Hospitalizaciones evitables por ACSC

\section{SATISFACCIÓN DEL USUARIO}

IV) EVALUACIÓN DE VARIOS COMPONENTES: Evaluación de varios componentes o dimensiones en el mismo estudio 